\title{
Moderate whisky consumption in combination with an evening meal reduces tryptophan availability to the brain but does not influence performance in healthy volunteers
}

\author{
C. Rob Markus ${ }^{1,2} *$, Aafje Sierksma ${ }^{3,4}$, Cees Verbeek $^{5}$, Jan J. M. van Rooijen ${ }^{5}$, Hamina J. Patel ${ }^{6}$, \\ A. Nico Brand ${ }^{7}$ and Henk F. J. Hendriks ${ }^{3}$ \\ ${ }^{1}$ University of Maastricht, Department of Experimental Psychology, PO Box 6200 MD Maastricht, The Netherlands \\ ${ }^{2}$ TNO Nutrition and Food Research, Department of Target Organ Toxicology, Zeist, The Netherlands \\ ${ }^{3}$ TNO Nutrition and Food Research, Department of Nutritional Physiology, Zeist, The Netherlands \\ ${ }^{4}$ University Medical Center Utrecht, Julius Center for Health Sciences and Primary Care, Utrecht, The Netherlands \\ ${ }^{5}$ TNO Nutrition and Food Research, Department of Food and Food Supplement Analysis, Zeist, The Netherlands \\ ${ }^{6}$ TNO Bibra International Limited, Surrey, UK \\ ${ }^{7}$ Utrecht University, Department of Health Psychology, Utrecht, The Netherlands
}

(Received 8 March 2004 - Revised 17 August 2004 - Accepted 17 August 2004)

\begin{abstract}
Brain serotonin (5-HT) synthesis is controlled by nutrients that influence the availability of plasma tryptophan (Trp) as compared with the sum of the other large neutral amino acids (LNAA; Trp:LNAA). Alcohol consumption is found to change mood and performance and this might well be due to alterations in the plasma Trp:LNAA ratio and brain 5-HT. In the present study, we tested whether whisky consumption as part of a meal may alter the plasma Trp:LNAA ratio and influence mood and performance in healthy volunteers. Twenty-four healthy male subjects participated in a within-subjects cross-over study. Subjects consumed whisky (125 ml; $40 \mathrm{~g}$ alcohol) or water $(125 \mathrm{ml})$ as part of a standard evening meal. Effects of whisky consumption were tested on mood and choice reaction time and blood samples were taken to measure changes in plasma amino acids, glucose and insulin. The plasma Trp:LNAA ratio showed a significant decline $2 \mathrm{~h}$ after whisky consumption of alcohol $(P<0 \cdot 001)$. No effects were found on choice reaction time or mood as compared with the control condition. The present findings reveal that whisky consumption alters available plasma Trp for uptake into the brain, whereas there were no effects on mood and performance.
\end{abstract}

Alcohol: 5-Hydroxytryptamine: Mood: Information processing: Cognition

It has frequently been demonstrated that alcohol consumption alters mood and performance. A majority of studies reveal a deterioration of information processing and performance 30-300 min after high doses $(0.3-1 \cdot 2 \mathrm{~g} / \mathrm{kg})$ of alcohol intake under a variety of different tasks (Maylor et al. 1992; for a review, see Koelega, 1995), whereas some have demonstrated cognitive improvement after moderate $(<0.3 \mathrm{~g} / \mathrm{kg})$ doses of alcohol intake (Maylor et al. 1987; Lloyd \& Rogers, 1997). For instance, in a study of Lloyd \& Rogers (1997), performance and mood improved even 90 and $150 \mathrm{~min}$ after the intake of $8 \mathrm{~g}$ alcohol (vodka) as part of a small meal. Particularly, the effects found after approximately $2 \mathrm{~h}$ are intriguing, since by that time breath alcohol concentration (BAC) should have returned almost to baseline levels. The authors explained the beneficial effects on performance by the 'calming' effects of alcohol. Although mood may influence performance, it remains questionable whether this constitutes a sufficient explanation, since the effects of alcohol on performance have also been mentioned in the absence of mood alterations (Liguori et al. 1999).

An additional mechanism mediating the effects of alcohol on mood and performance may be a change in the brain biochemical activity. Hence, there is supportive evidence that alcohol may act upon cerebral neurotransmitter activity (Eckardt et al. 1998) and a likely candidate may be the neurotransmitter brain 5-hydroxytryptamine (5-HT) that, among others, appears to be involved in the control of mood and cognitive performance (Maes \& Meltzer, 1995; Altman \& Normile, 1998). Brain 5-HT synthesis depends on the availability of its plasma precursor tryptophan (Trp) as compared with the other large neutral amino acids (LNAA) for which Trp competes for uptake into the brain (Fernstrom \& Wurtman, 1972; Curzon, 1985) and the

\footnotetext{
Abbreviations: BAC, breath alcohol concentration; 5-HT, 5-hydroxytryptamine (serotonin); LNAA, large neutral amino acids; POMS, Profile of Mood States; RT, reaction time; Trp, tryptophan.

* Corresponding author: Dr C. R. Markus, fax + 3143 3884199, email R.Markus@psychology.unimaas.nl
} 
plasma Trp:LNAA ratio is an accurate predictor of brain Trp and 5-HT alterations. The plasma Trp:LNAA ratio is controlled by food intake, either by the amount of carbohydrates as compared with protein (Fernstrom \& Wurtman, 1972) or by the amount of Trp in protein (Markus et al. 2000). Dietary changes in plasma Trp:LNAA are commonly found $2 \mathrm{~h}$ after intake and are accompanied by changes in brain 5-HT, mood and mental performance (Rosenthal et al. 1989; Bellisle et al. 1998; Markus et al. 1998, 2000, 2002). Since alcohol consumption is thought to change liver Trp pyrrolase (Badawy, 1996), the effect of alcohol on performance may be partly mediated by changes in plasma Trp:LNAA and brain 5-HT. In support of this assumption, ethanol intake has been found to influence brain 5-HT in animal studies (LeMarquand et al. 1994).

Although dietary changes in the plasma Trp:LNAA ratio may constitute an additional mechanism involved in the effects of macronutrients on mood and performance, no such combined behavioural studies have yet been conducted with alcohol consumption. Only a few studies have attempted to reveal whether ethanol consumption may have an effect on total plasma Trp concentrations (Badawy et al. 1987; Morgan \& Badawy, 2001) and only one has investigated the effect of ethanol on the Trp:LNAA ratio (Badawy et al. 1995). In this latter study, it was demonstrated that ethanol consumption decreases the Trp:LNAA ratio over a $2-3 \mathrm{~h}$ time interval. Yet, it remains unknown whether this is also found with a more ecologically valid alcohol consumption, in combination with a meal, and whether this also may be accompanied by changes in mood and performance.

The aim of the present study was to test whether whisky consumption in combination with a meal may alter the plasma Trp:LNAA ratio. Subjects participated in a within-subjects cross-over study in which they consumed whisky or water as part of a standard evening meal. At $2 \mathrm{~h}$ after consumption, blood samples were taken to measure the alcohol effects on plasma glucose, insulin and Trp:LNAA concentrations.

\section{Methods}

\section{Subjects}

Twenty-four male subjects aged 45-65 years (mean 52 (SD 5) years), all apparently healthy and non-smoking, were recruited from the TNO BIBRA International Ltd database of healthy human volunteers, by advertising in local newspapers and by leaflet drops to local residential areas. Subjects fulfilled the following inclusion criteria: consumption between ten and twenty-eight alcohol-containing beverages weekly (mean 16 (SD 5.72) units/ week), BMI between 20 and $35 \mathrm{~kg} / \mathrm{m}^{2}$ (mean BMI 26 (SD 3) $\mathrm{kg} / \mathrm{m}^{2}$ ) and no family history of alcoholism. Subjects were healthy as indicated by the values of the pre-study laboratory tests (2 weeks before onset of the study), their medical history and the physical examination by the medical investigator. A course of prescribed drug treatment within $28 \mathrm{~d}$ of the study start that had not been approved by the investigator was an exclusion criterion. One volunteer was withdrawn from the study due to a treatment-unrelated cause. The remaining twenty-three subjects finished the experiment successfully.

The study was conducted in accordance with the Declaration of Helsinki South Africa Revision 1996 and ICH Harmonized Tripartite Guideline for Good Clinical Practice. Approval to proceed with the present study was given by the Quality Assurance Unit (QAU) of the TNO Medical Ethical Committee, and all subjects provided written informed consent before participation.

\section{Procedure}

The experimental procedure was conducted according to a randomised, cross-over controlled design. On two experimental days, separated by 2 weeks, the subjects either received whisky (Famous Grouse Scotch Whisky, $40 \%$ (v/v) alcohol) or tap water (control) as part of an evening meal.

Four glasses $(125 \mathrm{ml}$ in total) of each beverage were consumed during the evening meal at TNO BIBRA. One glass was taken within $5 \mathrm{~min}$ before the evening meal, two glasses within 15 min during the meal and one glass within $5 \mathrm{~min}$ after the meal was completed (alcohol intake was $40 \mathrm{~g}$ within $25 \mathrm{~min}$ ). The evening meal contained approximately $4184 \mathrm{~kJ}(1000 \mathrm{kcal})$ and was made up of: fat $38 \%$ energy, protein $21 \%$ energy and carbohydrate $41 \%$ energy (calculated with the CompEat4; Nutrition Systems, Grantham, Lincolnshire, UK) (see Table 1).

On the experimental test day of each treatment condition, the subjects received an evening meal at 17.00 hours combined with either whisky or water. At $2 \mathrm{~h}$ after dietary intake (at 21.00 hours), a blood sample was taken and thereafter (21.15 hours) a psychometric test was conducted. In addition, before water and whisky consumption and

Table 1. Composition of the lunch boxes and evening meals by weight $(\mathrm{g})$ and percentage energy

\begin{tabular}{|c|c|c|c|c|c|c|c|}
\hline & \multicolumn{2}{|c|}{ Carbohydrate } & \multicolumn{2}{|c|}{ Protein } & \multicolumn{2}{|c|}{ Fat } & \multirow[b]{2}{*}{ Energy (kJ) } \\
\hline & Weight & \% Energy & Weight & \% Energy & Weight & \% Energy & \\
\hline $\begin{array}{l}\text { Lunch box } \\
\text { Bread roll, butter and cheese } \\
\text { Ham, cucumber, lettuce } \\
\text { Crisps }(25 \mathrm{~g}), \text { yoghurt } \\
\text { Orange juice }(200 \mathrm{~g})\end{array}$ & 161 & 47 & 37 & 11 & 59 & 42 & 5371 \\
\hline $\begin{array}{l}\text { Evening meal } \\
\text { Chicken and bacon risotto } \\
\text { Tart au chocolate }\end{array}$ & 104 & 40 & 54 & 21 & 44 & 39 & 4276 \\
\hline
\end{tabular}


repeatedly $(30,60,90$ and $180 \mathrm{~min})$ after whisky consumption blood alcohol levels were measured by breath analysis (Alcoholtest 7410; Dräger Nederland B.V., Zoetermeer, The Netherlands). For the psychometric test the subject was taken to a room, seated in front of a computer screen and instructed about the experiment. The subject was then exposed to a computer-assisted battery of psychometric tests, all integrated into the research software package MINDS (Brand, 1999). The battery consisted of a version of the Profile of Mood States (POMS; McNair et al. 1971) and a Sternberg's memory-scanning task (Sternberg, 1969). The subjects spent approximately 4 min on the POMS and 12 min on the memory-scanning task. At 1 week before the commencement of the present study, all volunteers were given a practice session in order to familiarise them with the procedures.

There was a $3 \mathrm{~d}$ alcohol-free period before both experimental days to prevent possible carry-over effects and the subjects were required to remain alcohol-free during the experiment. On both test days, lunch boxes were supplied, which had to be consumed $5 \mathrm{~h}$ before testing, in order to standardise food intake. The subjects were instructed to consume and register all the food items provided with the lunch boxes and the food diary was documented and evaluated by the researcher to ascertain compliance. In addition, the same amount of consumed items was given to the subjects on the second test condition. Within $5 \mathrm{~h}$ before the test, the subjects were requested only to consume caffeine- and nutrient-free drinks and the lunch box. Evening meals and lunch boxes were prepared by a local caterer using the same source of ingredients throughout (see Table 1 for the composition of lunch boxes and evening meals).

\section{Profile of Mood States}

Changes in mood were measured using the POMS questionnaire (McNair et al. 1971), offered at the computer screen on a five-point interval scale ranging from 'strongly disagree' to 'strongly agree'. The POMS comprises adjectives for five different subscales for mood. The subscale Anger (range 7-35), Depression (range 8-40), Fatigue (range 6-30) and Tension (range 6-30) refer to a negative mood state, whereas the subscale Vigour (range 5-25) concerns a positive mood.

\section{Cognitive performance}

Cognitive performance refers to the observable outcome of mental computations involved in the perception, recognition and encoding of a stimulus (input level) as well as the preparation and organisation of a response (output level). A computerised Sternberg memory-scanning task (Sternberg, 1969) was used as a cognitive performance test since this task allows for subtracting different stages of mental processing. This task consisted of four subtasks, corresponding to memory sets of one, two, three and four different consonants. Each subtask started with a presentation on the computer screen of the particular set to be memorised, for as long as each subject needed, after which sixty trials followed. In thirty trials the presented letter belonged to the memorised set; in the other thirty trials this was not the case. Every first ten trials in each subtask were used for practice and, therefore, not used in the analysis. In each trial the probe letter was presented at the centre of the screen for $1 \mathrm{~s}$ (machine-paced; with an interval range of $1 \mathrm{~s}$ ). The order of presentation of the sixty letters was randomised. The subjects were instructed to decide as quickly and accurately as possible whether the presented letter did or did not belong to the memory set by pressing a red thumb-key button (No) or a green thumbkey button (Yes).

\section{Biochemical analyses}

Blood samples were collected in a tube containing EDTA (Vacutainer Systems; Becton Dickinson, Plymouth, Devon, UK) for amino acids analysis and in a tube (Vacutainer Systems) containing gel and clot activator for insulin and glucose analysis. The EDTA tube was centrifuged at $2000 \mathrm{~g}$ for $30 \mathrm{~min}$ at $20^{\circ} \mathrm{C}$ within $15 \mathrm{~min}$ of sampling. The tube containing gel and clot activator was centrifuged at $2000 \mathrm{~g}$ for $10 \mathrm{~min}$ at $4^{\circ} \mathrm{C}$ between 15 and $30 \mathrm{~min}$ after collection. Plasma and serum were directly stored at $-80^{\circ} \mathrm{C}$ until analysis.

For the determination of amino acids in plasma, a reproducible, fully automated, method was used based on cation exchange chromatography and post-column derivatisation with ninhydrin.

Plasma samples were deproteinised with 5-sulfosalicylic acid. Norvaline was used as an internal standard. Separation of the amino acids was performed with a stepwise gradient of four lithium citrate buffers on a BTC-2710 cation exchange resin. Column dimensions were $180 \times 3.2 \mathrm{~mm}$. After post-column derivatisation, amino acids were detected at $570 \mathrm{~nm}$. The plasma Trp ratio was ultimately calculated by dividing the plasma Trp concentration by the sum of the other LNAA, i.e. valine, isoleucine, leucine, tyrosine and phenylalanine.

Insulin concentrations in plasma were assayed by using a standard enzyme radioimmunoassay kit (Tosoh Corporation, Tokyo, Japan).

Glucose concentrations were assayed in plasma by enzymic antidiuretic hormone using a standard hexokinase kit (Roche Diagnostic, Mannheim, Germany).

\section{Experimental design and statistical analysis}

The main research questions formulated in the introduction were analysed by means of multi- and univariate ANOVA using the general linear model (SPSS 7.5 for Windows; SPSS Inc., Chicago, IL, USA). The design of the present study included 'Treatment' (whisky $v$. water) as the within-subjects factor. The dependent variables were the plasma Trp:LNAA ratio and glucose and insulin concentrations; the scores on the different scales of the POMS and reaction time (RT) on (the different levels of) the Sternberg task. For the effect of 'Treatment' (whisky $v$. water) on the five mood scales of the POMS, as well as on RT and the amount of errors during the different subtasks of the Sternberg's performance test, different multivariate ANOVA were performed. For the scores on the four different 
consecutive subtasks of the memory-searching task (memory sets of one, two, three and four consonants) the multivariate analysis was performed with first- and second-order polynomial contrasts (linear and quadratic effects). Only significant multivariate results revealed by these procedures were further examined by univariate tests. Huynh-Feldt or Greenhouse-Geisser corrected $P$ values, their corresponding epsilons as well as the original, i.e. uncorrected, degrees of freedom were reported when the sphericity assumption was not met. All statistics were evaluated at a significance level of $5 \%$ (two-tailed). Data are reported as mean values and SD.

\section{Results}

\section{Blood alcohol assessment}

To estimate BAC, a one-way ANOVA with 'Time' (30, 60, 90 and $180 \mathrm{~min}$ after whisky consumption) as the withinsubjects factor was conducted on BAC after whisky consumption. Analysis revealed a significant effect of 'Time', meaning that BAC significantly declined over time after whisky consumption. BAC increased from zero before treatment to 0.5 (SD $0 \cdot 15) \mathrm{g} / \mathrm{l}$ at $30 \mathrm{~min}$ after whisky consumption, which significantly declined to 0.44 (SD 0.13) g/l after $60 \mathrm{~min}, 0.36$ (SD 0.12) g/l after $90 \mathrm{~min}$ and $0 \cdot 14(\mathrm{SD} 0 \cdot 12) \mathrm{g} / \mathrm{l}$ after $180 \mathrm{~min}$ since drinking.

\section{Plasma glucose and insulin concentration}

In order to test the effects of whisky consumption on plasma glucose and insulin concentrations $2 \mathrm{~h}$ after consumption, we conducted paired-sample $t$ tests with 'Treatment' (whisky $v$. water) as the within-subjects factor on plasma samples. Analysis revealed a significant difference in glucose concentrations between treatment conditions $(t-3.039$; df $22 ; P=0.006)$. As shown in Fig. 1 (a), plasma glucose concentrations were significantly lower $2 \mathrm{~h}$ after whisky consumption (50 (SD 0.95) mU/l) as compared with after water consumption (59 (SD 0.95) mU/l). No differences were found between whisky and water consumption on plasma insulin concentrations $(P>0.4)$.

\section{Plasma tryptophan:large neutral amino acids ratio}

In order to test the effect of whisky consumption on the plasma Trp:LNAA ratio, we conducted a paired sample $t$ test on plasma amino acid concentrations measured $2 \mathrm{~h}$ after whisky and water consumption. Analysis revealed a significant difference between whisky and water consumption $(t 4.60$; df 22; $P<0.0001)$. As shown in Fig. 1 (b), the plasma Trp:LNAA ratio declined after whisky consumption (0.058 (SD 0.009)) as compared with after water consumption (0.064 (SD 0.008)).

To test the effect of whisky consumption on plasma total Trp concentrations, we also performed two $t$ test analyses on plasma Trp concentration and on the sum of the competitor LNAA $2 \mathrm{~h}$ after whisky and water consumption. As shown in Table 2, analysis revealed a significant decline in plasma total Trp concentrations $2 \mathrm{~h}$ after alcohol consumption (35 (SD 6) $\mu \mathrm{mol} / \mathrm{l}$ ) as compared with after
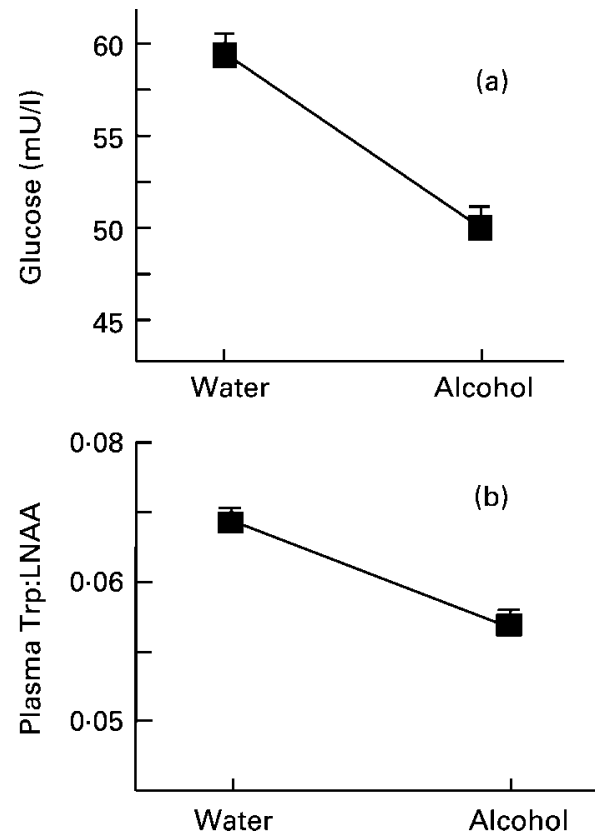

Fig. 1. (a) Plasma glucose concentrations were significantly lower $2 \mathrm{~h}$ after alcohol consumption (50 (SD 0.95) $\mathrm{mU} / \mathrm{l}$ ) as compared with after water consumption (59 (SD 0.95) mU/l) $(t-3.039$; df 22; $P=0.006$ ). (b) There was a significant reduction in plasma tryptophan:large neutral amino acids (Trp:LNAA) ratio $2 \mathrm{~h}$ after alcohol consumption (0.058 (SD 0.009)) as compared with after water consumption (0.064 (SD 0.008)) ( $t 64$; df 22; $P=0.004)$.

water consumption (41 (SD 6) $\mu \mathrm{mol} / \mathrm{l})$ ( $t$ 4.912; df 22; $P<0.0001$ ), whereas there were no differences in concentrations of the sum of the competitor LNAA $(P>0 \cdot 18)$.

\section{Mood (Profile of Mood States)}

In order to see whether whisky consumption may influence mood, we performed a multivariate ANOVA with 'Treatment' (whisky $v$. water) as the within-subjects factor on the scores of the five different mood scales. However, multivariate analysis did not reveal a significant effect of 'Treatment' $(P>0 \cdot 4)$.

\section{Cognitive performance}

To see whether alcohol consumption could affect performance, we performed an ANOVA on the mean RT across all four subtasks, taken as an overall measure of performance efficiency. ANOVA with 'Treatment' (whisky $v$. water) as

Table 2. Effect of whisky on plasma concentrations of tryptophan (Trp) and the sum of its competitors (large neutral amino acids; LNAA) $2 \mathrm{~h}$ after intake

(Mean values and standard deviations)

\begin{tabular}{|c|c|c|c|c|c|c|c|}
\hline \multicolumn{4}{|c|}{$\operatorname{Trp}(\mu \mathrm{mol} / \mathrm{l})$} & \multicolumn{4}{|c|}{ Sum of LNAA } \\
\hline \multicolumn{2}{|c|}{ Whisky } & \multicolumn{2}{|c|}{ Water } & \multicolumn{2}{|c|}{ Whisky } & \multicolumn{2}{|c|}{ Water } \\
\hline Mean & SD & Mean & SD & Mean & SD & Mean & SD \\
\hline 35 & 6 & $41^{*}$ & 6 & 627 & 115 & 645 & 105 \\
\hline
\end{tabular}

${ }^{*}$ Mean value was significantly different from that after whisky $(P<0.0001)$. 
the within-subjects factor did not reveal a significant effect $(P>0 \cdot 8)$.

It was also explored whether an increase in RT as a function of memory load was influenced by whisky consumption as compared with water consumption. This slope - the rate at which RT increases with memory load - is interpreted by Sternberg (1975) as an indicator of the time needed for scanning an item in short-term memory. Multivariate ANOVA with 'Treatment' (whisky $v$. water) and 'Memory-Load' (one, two, three and four consonants) as within-subjects factors only revealed an effect of 'Memory-Load' $(F(3,20) 49.43 ; P<0.0001)$, indicating that RT increases as a function of memory load regardless of treatment condition. There was no interaction effect of 'Treatment' by 'Memory-Load' $(P>0 \cdot 6)$.

\section{Discussion}

In the present study, we tested whether moderate alcohol consumption could alter the plasma Trp:LNAA ratio and change mood and cognitive performance in healthy volunteers. Although findings revealed a significant effect of whisky consumption on the plasma Trp:LNAA ratio, this was not accompanied with significant changes in mood or choice RT.

\section{Effect of whisky on blood alcohol concentration}

Consumption of whisky containing $40 \mathrm{~g}$ (approximately $0.5 \mathrm{~g} / \mathrm{kg}$ ) of alcohol in combination with an evening meal clearly increased BAC by $0.51 \mathrm{~g} / \mathrm{l}$ at $30 \mathrm{~min}$ after consumption and by $0 \cdot 14 \mathrm{~g} / \mathrm{l}$ at $180 \mathrm{~min}$ after consumption. These alterations in BAC are comparable with those reported after exclusive comparable amounts of alcohol consumption (Koelega, 1995; Hendriks et al. 1998; Sierksma et al. 2002).

\section{Effects of alcohol on the plasma amino acids}

Glucose and tryptophan:large neutral amino acids ratio. The results of the present study revealed that the consumption of four glasses of whisky ( $40 \mathrm{~g}$ alcohol) with an evening meal causes a significant decline in the plasma Trp:LNAA ratio, as compared with after water consumption, $2 \mathrm{~h}$ after consumption. In addition, plasma glucose concentration declined $2 \mathrm{~h}$ after whisky consumption, whereas no differences were found for insulin concentrations. This decline in glucose concentration most probably represents a hypoglycaemic response due to an insulin-mediated glucose uptake. Hence, in previous studies increased plasma glucose levels are generally found within 30-60 min after alcohol consumption which decline below baseline approximately after $>1.5 \mathrm{~h}$ (Badawy et al. 1987; Morgan \& Badawy, 2001). This effect of whisky consumption on the plasma Trp:LNAA ratio in the present study is consistent with previous findings of a decline in plasma Trp:LNAA after single ethanol ingestion in fasting male volunteers (Badawy et al. 1995). In this latter study, the consumption of $0.8 \mathrm{~g}$ ethanol/kg significantly decreased the plasma Trp:LNAA by $17 \%$ as compared with control subjects $2 \mathrm{~h}$ after consumption.
Taking into account the effects of alcohol on short-term alterations in blood glucose, it is not clear why alcohol consumption is accompanied by a significant decline in the plasma Trp:LNAA ratio. Hence, an increase in plasma glucose concentration constitutes a necessary condition for carbohydrate ingestion to cause an insulinmediated increased uptake of the LNAA into the skeletal muscles, causing a consequent rise in the plasma Trp:LNAA ratio and brain 5-HT (Fernstrom \& Wurtman, 1972; Curzon, 1985). In a previous study of Badawy et al. (1995), the decline in the plasma Trp:LNAA ratio after alcohol consumption has been attributed to an enhanced hepatic Trp pyrrolase, since ethanol intake was accompanied by a decline in both free and total (free and albumin-bound) Trp concentration. Hence, particularly when alcohol is consumed in combination with solid food, first-pass metabolic effects such as increases in hepatic Trp pyrrolase may be most pronounced (Eckardt et al. 1998). The present results revealed a significant decline in plasma Trp concentrations $2 \mathrm{~h}$ after alcohol consumption as compared with after water consumption, whereas there were no differences in the sum of concentrations of the competitor LNAA. These findings support the assumption that Trp concentration, and subsequently the plasma Trp:LNAA ratio, is reduced by enhanced Trp pyrrolase. However, the exact mechanism by which alcohol consumption, whether or not combined with food intake, enhances first-pass metabolic processes is still poorly understood and needs further investigation.

\section{Effects of alcohol on mood and performance}

It was expected that mood as well as choice RT during memory scanning might be influenced by alcohol consumption as a function of alterations in plasma Trp:LNAA. However, even though the plasma Trp:LNAA ratio had significantly declined $2 \mathrm{~h}$ after whisky consumption, no effects on mood or choice RT were detected. On the first hand, the present data do not seem to confirm previous findings on the effects of alcohol consumption on mood and performance during comparable demanding task performance in which comparable alterations in BAC have been demonstrated (Maylor et al. 1987; Koelega, 1995; Lloyd \& Rogers, 1997). However, we should be careful in generalising these present findings as evidence for a lack of a neurocognitive effect of alcohol, since we only used one test for cognitive performance and, in comparison with Lloyd \& Rogers (1997), we also included a diet that differed in energy constitution and was given at a different time of day.

The absence of an effect on mood and performance seems to be in compliance with the limited change in the plasma Trp:LNAA ratio. Hence, in depletion studies, the most clear behavioural effects of Trp depletion have been reported after a decline of $50 \%$. In addition, a current $10 \%$ decline in plasma Trp:LNAA after consuming modest amounts of alcohol may not be sufficient enough to cause meaningful brain and behavioural alterations. However, the present findings may have particular relevance for clinical populations. Hence, this decline in plasma Trp:LNAA may be more profound in problem drinkers, using larger amounts of alcohol for a longer 
period of time, and may be partly responsible for cognitive and emotional decline as often demonstrated in these clinical populations.

\section{Conclusion}

Modest alcohol consumption in the present study significantly declines the plasma Trp:LNAA ratio $2 \mathrm{~h}$ after consumption without any effect on mood and choice RT. These findings challenge assumptions that modest alcohol consumption in healthy subjects could change mood and information processing by alterations in brain Trp and 5-HT concentrations. However, the present findings may be relevant in problem drinkers in which the effects of long-lasting consumption of larger amounts of alcohol on brain 5-HT may be more pronounced and may have severe emotional and behavioural consequences.

\section{Acknowledgements}

We acknowledge all those involved in the conduct of the present study and thank the volunteers for their enthusiastic participation. The research described in the present paper was funded by the Dutch Foundation for Alcohol Research (SAR).

\section{References}

Altman HJ \& Normile HJ (1998) What is the nature of the role of the serotonergic nervous system in learning and memory: prospects for development of an effective treatment strategy for senile dementia. Neurobiol Aging 9, 627-638.

Badawy AAB (1996) Tryptophan metabolism and disposition in relation to alcohol and alcoholism. Adv Exp Med Biol 398, 75-82.

Badawy AAB, Morgan CJ, Lovett JWT, Bradley DM \& Thomas DR (1995) Decrease in circulating tryptophan availability to the brain after acute ethanol consumption by normal volunteers: implications for alcohol-induced aggressive behaviour and depression. Pharmacopsychiatry 28, 93-97.

Badawy AAB, Morgan CJ, Thomas DR \& Lovett JWT (1987) The acute effects of ethanol on the serum concentrations of tryptophan and other constituents in fasting normal male volunteers. Ann Clin Biochem 24, S1-63-S1-65.

Bellisle F, Blundell JE, Dye L, Fantino M, Fletcher RJ, Lambert J, Roberfroid M, Specter S, Westenhöfer J \& Westerterp-Plantenga MS (1998) Functional food science and behaviour and psychological functions. Br J Nutr 80, S173-S193.

Brand AN (1999) MINDS: tool for research in health psychology and neuropsychology. In Cognitive Ergonomics, Clinical Assessment and Computer-assisted Learning, pp. 155-168 [BPLM den Brinker, PJ Beek, AN Brand, FJ Maarse and LJM Mulder, editors]. The Netherlands: Swets \& Zeitlinger.

Curzon G (1985) Effects of food intake on brain transmitter amine precursors and amine synthesis. In Psychopharmacology and Food, pp. 59-70 [M Sandler and T Silverstone, editors]. Oxford: Oxford University Press.

Eckardt MJ, File SE, Gessa GL, Grant KA, Guerri C, Hoffman PL, Kalant H, Koob GF, Li TK \& Tabakoff B (1998) Effects of moderate alcohol consumption on the central nervous system. Alcohol Clin Exp Res 22, 998-1040.

Fernstrom JD \& Wurtman RJ (1972) Brain serotonin content: physiological regulation by plasma neutral amino acids. Science 178, 414-416.

Hendriks FJ, Veenstra J, van Tol A, Groener JEM \& Schaafsma G (1998) Moderate doses of alcoholic beverages with dinner and postprandial high density lipoprotein composition. Alcohol Alcohol 4, 403-410.

Koelega HS (1995) Alcohol and vigilance performance: a review. Psychopharmacology 118, 233-249.

LeMarquand D, Pihl RO \& Benkelfat C (1994) Serotonin and alcohol intake, abuse, and dependence: findings of animal studies. Biol Psychiatry 36, 395-421.

Liguori A, D'Agostino RB, Dworkin SI, Edwards D \& Robinson JH (1999) Alcohol effects on mood, equilibrium and simulated driving. Alcohol Clin Exp Res 23, 815-821.

Lloyd HM \& Rogers PJ (1997) Mood and cognitive performance improved by a small amount of alcohol given with a lunchtime meal. Behav Pharmacol 8, 188-195.

McNair DM, Lorr M \& Dropleman LF (1971) EITS Manual Profile of Mood States. San Diego, CA: Educational and Testing Service.

Maes M \& Meltzer H (1995) The serotonin hypothesis of major depression. In Psychopharmacology: the Fourth Generation of Progress, pp. 933-944 [FE Bloom and DJ Kupfer, editors]. New York: Raven Press.

Markus CR, Olivier B \& de Haan EHF (2002) Whey protein rich in alpha-lactalbumin increases the plasma Trp/LNAA ratio and improves cognitive performance in stress-vulnerable subjects. Am J Clin Nutr 75, 1051-1056.

Markus CR, Olivier B, Panhuysen G, Van der Gugten J, Alles M, Tuiten A, Westenberg HGM, Fekkes D \& Koppeschaar H (2000) The bovine protein alpha-lactalbumin increases the plasma Trp/LNAA ratio, and in vulnerable subjects raises brain serotonin activity and decreases cortisol and mood under stress. Am J Clin Nutr 71, 1536-1544.

Markus CR, Panhuysen G, Tuiten A, Koppeschaar H, Fekkes D \& Peters M (1998) Does carbohydrate-rich, protein-poor food prevent a deterioration of mood and cognitive performance of stress-prone subjects when subjected to a stressful task? Appetite 31, 49-65.

Maylor EA, Rabbitt PMA, James GH \& Kerr SA (1992) Effects of alcohol, practice and task complexity on reaction time distributions. Q J Exp Psychol 44A 119-139.

Maylor EA, Rabbitt PMA, Sahgal A \& Wright C (1987) Effects of alcohol on speed and accuracy in choice reaction time and visual search. Acta Psychol (Amst) 65, 147-163.

Morgan CJ \& Badawy AAB (2001) Alcohol-induced euphoria: exclusion of serotonin. Alcohol Alcohol 36, 22-25.

Rosenthal NE, Genhart MJ, Caballero B, Jacobsen FM, Skwerer RG, Coursey RD, Rogers S \& Spring B (1989) Psychobiological effects of carbohydrate- and protein-rich meals in patients with seasonal affective disorder and normal controls. Biol Psychiatry 25, 1029-1040.

Sierksma A, van der Gaag S, van Tol A, James RW \& Hendriks HFJ (2002) Kinetics of HDL cholesterol and paraoxonase activity in moderate alcohol consumers. Alcohol Clin Exp Res 26, 1430-1435.

Sternberg S (1969) Memory-scanning: mental processes revealed by reaction-time experiments. Am Sci 57, 421-457.

Sternberg S (1975) Memory scanning: new findings and current controversies. Q J Exp Psychol 27, 1-32. 